\title{
High-Precision Contour-Tracking Control of Ethernet-Based Networked Motion Control Systems
}

\author{
Xiang $\mathrm{Wu}^{*, * *}$ Non-member, Hui Dong*a) Non-member \\ Jinhua She ${ }^{* *}$ Senior Member, $\mathrm{Li} \mathrm{Yu}^{*} \quad$ Non-member
}

(Manuscript received Aug. 17, 2018, revised June 27, 2019)

\begin{abstract}
This paper focuses on the high-precision contour-tracking control of an Ethernet-based networked motion control system (ENMCS) with short time-varying delays and repetitive tasks. The delays and the aperiodic exogenous disturbance are the main factors that deteriorate the tracking performance. An integrated control approach is devised, which consists of three parts: a disturbance observer for rejecting the delay and disturbance, an iterative learning controller for achieving perfect individual-axes tracking, and a cross-coupled controller for further improving the contour-tracking performance. In addition, a fuzzy regulator is proposed in the iteration domain to adjust the contour-controller gains so as to smooth iteration processes of individual axes. Comparative simulations and experiments are carried out to verify the feasibility and effectiveness of the approach.
\end{abstract}

Keywords: networked motion control system (NMCS), time-varying delays, iterative learning control, aperiodic disturbance, contour tracking control

\section{Introduction}

In recent years, intelligent manufacturing has become a new trend in industry, and the development of motion control systems, which are one of the core components, also has made great progress. Internet technologies have been used to overcome the complexity in wiring, difficulty in maintenance, poor in expansibility, and other weaknesses of the conventional motion control systems. CAN, Ethernet POWERLINK, Ethernet/IP, EtherCAT, and other fieldbuses are successfully used for the intelligent manufacturing ${ }^{(1)-(5)}$. But a general-purpose Ethernet has the advantages of low cost, openness, and universality. However, some new problems need to be solved for the use of a general-purpose Ethernet in a motion control system. One of them is a network-induced delay. Such a delay may seriously degrades system performance ${ }^{(6)(7)}$. Thus, the introduction of a general-purpose Ethernet into motion control systems is a challenging problem in both academic and industrial fields.

A switched system ${ }^{(8)}$, robust control ${ }^{(9)}$, a Smith predictor $^{(10)}$, the model predictive control ${ }^{(11)}$, a communication disturbance observer $(\mathrm{CDOB})^{(12)}$, and other approaches have been presented to deal with a delay issue. Among them, the disturbance-observer-based control (DOBC) approach does not need a trade-off between the system stability and control performance ${ }^{(13)}$. It also has a potential for handling a timevarying delay.

a) Correspondence to: Hui Dong. E-mail: hdong @ zjut.edu.cn

${ }^{*}$ School of Information Engineering, Zhejiang University of Technology

Hangzhou, 310023, China

** School of Computer Science, Tokyo University of Technology

Hachioji, Tokyo 192-0982, Japan
Motion control of multi-axis systems is mainly classified into three types: positioning control, tracking control, and contouring control ${ }^{(14)}$. Contouring control is the most critical task $^{(15)}$, and has attracted a lot of attentions ${ }^{(16)-(18)}$. In order to suppress the contour error directly, Koren proposed the crosscoupled control (CCC) approach ${ }^{(19)}$, which has been proved to be effective. Since cutting, carving, welding, and many other motion control tasks in manufacturing are periodic, learning control is especially effective for those tasks ${ }^{(20)-(24)}$. Wu et al. applied iterative learning control (ILC) to achieve perfect contour following ${ }^{(21)}$, but it was not concerned with the tracking performance of each axis. Kuo et al. explored the use of a fuzzy-logic-based reinforcement ILC for the suppression of exogenous periodic disturbances to reduce the tracking error of each axis ${ }^{(22)}$. Barton and Alleyne designed a controller, which integrated individual-axis feedback control, individual-axis ILC, and cross-coupled ILC, to reduce both the tracking and contour errors ${ }^{(23)}$. Ling et al. modified the controller of (23) by introducing a position-domain control method to solve the problem of nonlinear contour tracking ${ }^{(24)}$. Even though aperiodic disturbances widely exist in real systems, they were not considered in previous studies. However, they may seriously deteriorate system performance.

The DOBC method is an effective way to reject aperiodic disturbances and uncertainties of a control system ${ }^{(25)-(28)}$, because it ingeniously utilizes disturbance information. Chen and Moore proposed a disturbance observer (DOB) in the iteration domain to attenuate an unknown aperiodic disturbance $^{(25)}$. Sun and Li designed a DOB-based ILC scheme for a class of systems with mismatched aperiodic disturbances ${ }^{(26)}$. Yu et al. devised an improved equivalentinput-disturbance (EID) approach to enhancing disturbancerejection performance of a repetitive-control system $^{(27)}$. 
Sayem et al. proposed an extended state observer (ESO)based repetitive control to compensate for periodic signals, aperiodic disturbances, and uncertainties of a plant ${ }^{(28)}$. The simple structure of the $\mathrm{ESO}^{(29)}$ makes it easy to be applied in control practice and disturbance rejection ${ }^{(30)(31)}$. On the other hand, when ILC is solely used for tracking control, the tracking errors are usually big in the first few iteration trials. Thus, the estimations of contour errors, which are calculated according to the tracking errors, are inaccurate at the beginning stage of control. Fixed gains of cross-coupled controller may also result in over compensation for individual axes. This deteriorates the tracking performance. Adjusting the gains according to the control result might be a possible solution to these problems, and fuzzy control is a possible way for this purpose $^{(32)(33)}$.

This paper discusses the use of a general-purpose Ethernet for a networked motion control system (ENMCS). First, ESO-based ILC for the controllers of individual axes are designed for tracking control, in which the ESO is used to suppress aperiodic disturbances containing those caused by delays. Then, a proportional-derivative (PD)-CCC is used to further improve the precision of contour tracking. A fuzzy regulator is used in the iteration domain to adjust gains of the PD-CCC so as to smooth iteration processes of each axes. The contributions of this paper are threefold:

(1) A new structure of an NMCS is presented and implemented based on a general-purpose Ethernet. This provides a simple frame, low cost, good universality, easy connectivity to the Internet, and other benefits. The control scheme and the configuration of the new system are simple. The system is easy to implement in an industrial process;

(2) The effect of time-varying delays is analyzed. The delays is modeled as a bounded disturbance. An aperiodic disturbance, which contains the one caused by the delays, is discussed for the ENMCS; and

(3) Iteration-domain fuzzy PD-CCC (IDFPD-CCC) is devised to further improve the control performance of contour tracking and to maintain the control performance of individual-axis tracking.

The remainder of this paper is organized as follows. Section 2 describes the ENMCS experimental setup and the network-induced delays. Section 3 explains the design of an ESO-based ILC controller. Section 4 devises a contour controller. Section 5 verifies the feasibility and effectiveness of the new system through simulations and experiments. Finally, Section 6 gives conclusion remarks.

\section{Ethernet-based Networked Motion Control System}

An experimental ENMCS (Fig. 1) contains a PC, an interface board, two servo systems, and a three-axis mechanical arm. The PC is a control center that contains control algorithms and is connected to the interface board through a general-purpose Ethernet. The interface board receives data from the PC using the TCP/IP protocol and sends them to servo systems using the CANopen protocol. The servo systems drive two AC motors, which are installed in the mechanical arm. The interface board and the servo systems are connected by a CAN bus. The control procedure has three

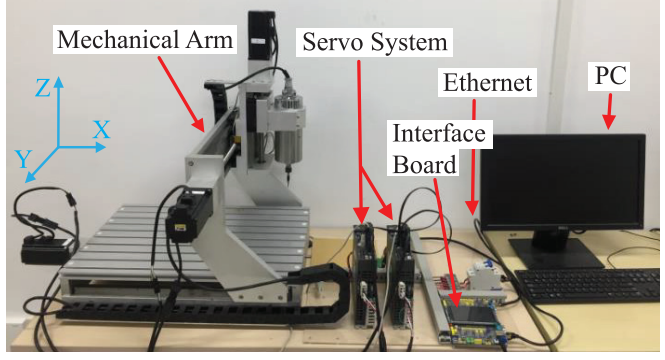

Fig. 1. Experimental setup of the ENMCS

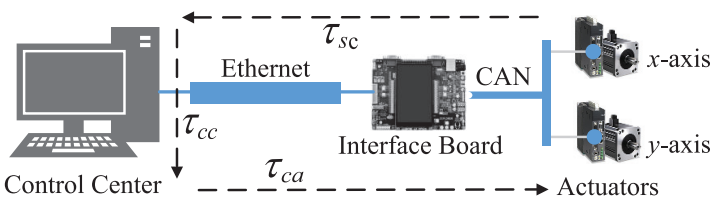

Fig. 2. Time-varying delays in the ENMCS

steps:

1. The control center discretizes a reference trajectory into discrete points, calculates control inputs, and sends them to the interface board in a real-time fashion.

2. The interface board reads the inputs using the TCP/IP protocol, encapsulates them using the CANopen protocol, and transfers them to the servo systems.

3. The servo systems receive the inputs and drive the motors. The positions are fed back to the PC through the communication network at every sampling instant.

The transfer functions of the AC servo systems in this study are

$$
P_{i}(s)=\frac{R_{i}(s)}{U_{i}(s)}=\frac{b_{i}}{s^{2}+a_{i 2} s+a_{i 1}}, \quad i=x, y, \cdots \cdots \cdots
$$

where $i=x(=y)$ is the one for $x$ - $(y-)$ axis; $U_{i}(s)$ is the control input; $R_{i}(s)$ is the output; $a_{i 1}, a_{i 2}$, and $b_{i}$ are constant parameters; and $U_{i}(s)$ and $R_{i}(s)$ are the transforms of $u_{i}(t)$ and $r_{i}(t)$, respectively.

A state-space model of $i$-axis is

$$
\left\{\begin{array}{l}
\dot{v}_{i 1}(t)=v_{i 2}(t) \\
\dot{v}_{i 2}(t)=-a_{i 1} v_{i 1}(t)-a_{i 2} v_{i 2}(t)+b_{i} u_{i}(t)+d_{i}(t), \cdots \cdots \\
r_{i}(t)=v_{i 1}(t)
\end{array}\right.
$$

where $t \in\left[\begin{array}{ll}0 & T\end{array}\right]$ and $T$ is the iteration period, $v_{i 1}(t)$ and $v_{i 2}(t)$ are system states, and $d_{i}(t)$ is a bounded aperiodic exogenous disturbance.

Delays in the control system are recorded in the interface board. The time delay of the system, $\tau_{k}$, has three parts (Fig. 2): a computation delay of the PC, $\tau_{c c}$, a transmission delay from the PC to the servo systems, $\tau_{c a}$, and a feedback transmission delay from the sensors to the PC, $\tau_{s c}$. So,

$$
\tau_{k}=\tau_{c c}+\tau_{c a}+\tau_{s c}
$$

Assume that the delay is not larger than one sampling period, $h$, that is, $0 \leq \tau_{k} \leq h$. The sensor is set to be time-driven, and the controller and actuators are event-driven. Then, the control input of the $i$-axis, $u_{i}(t)$, is ${ }^{(6)}$

$$
u_{i}(t)= \begin{cases}u_{i}\left(t_{k-1}\right), & t \in\left(t_{k}, t_{k}+\tau_{k}\right], \\ u_{i}\left(t_{k}\right), & t \in\left(t_{k}+\tau_{k}, t_{k+1}\right]\end{cases}
$$




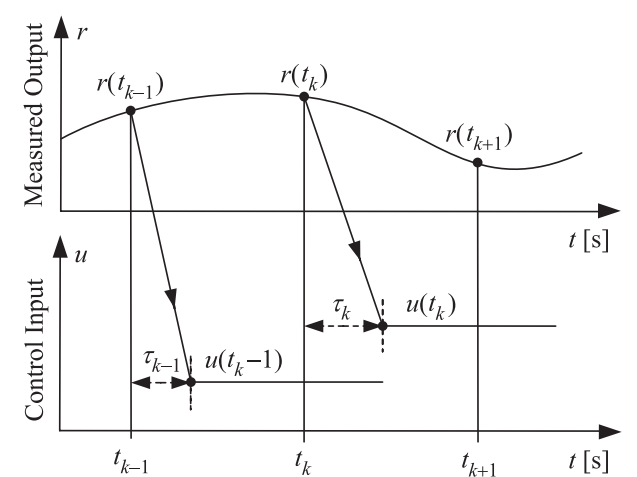

Fig. 3. Timing diagram of the ENMCS with timevarying delays of $i$-axis $(i=x, y)$

as shown in Fig. 3, where $t_{k}$ is the sampling time $k, t_{k+1}=$ $t_{k}+h, 1 \leq k<N$, and $N=T / h$.

Taking $\tau_{k}$ into account and combining (2) and (4) give

$$
\left\{\begin{array}{l}
v_{i 1}(k+1)=v_{i 1}(k)+h v_{i 2}(k), \\
v_{i 2}(k+1)=e^{-a_{i 2} h} v_{i 2}(k)+\frac{a_{i 1}}{a_{i 2}}\left(e^{-a_{i 2} h}-1\right) v_{i 1}(k) \\
\quad-\frac{b_{i}}{a_{i 2}}\left(e^{-a_{i 2}\left(h-\tau_{i k}\right)}-1\right) u_{i}(k) \\
\quad-\frac{b_{i}}{a_{i 2}}\left(e^{-a_{i 2} h}-e^{-a_{i 2}\left(h-\tau_{i k}\right)}\right) u_{i}(k-1)+d_{i}(k), \\
r_{i}(k)=v_{i 1}(k) .
\end{array}\right.
$$

Linearizing (5) yields

$$
\left\{\begin{aligned}
v_{i 1}(k+1) & =v_{i 1}(k)+h v_{i 2}(k), \\
v_{i 2}(k+1) & =v_{i 2}(k)+h\left[-a_{i 1} v_{i 1}(k)-a_{i 2} v_{i 2}(k)+b_{i} u_{i}(k)\right. \\
& \left.+\frac{b_{i} \tau_{i k}}{h}\left(u_{i}(k-1)-u_{i}(k)\right)+d_{i}(k)\right], \\
r_{i}(k) & =v_{i 1}(k),
\end{aligned}\right.
$$

where modeling error is contained in the disturbance $d_{i}(k)$. Then, the delay is modeled to be $d_{i \tau}(k)=\frac{b_{i} \tau_{i k}}{h}\left(u_{i}(k-1)-u_{i}(k)\right)$, which is treated as a part of the overall disturbance. Thus, the system is simplified to be a delay-free one. Note that the disturbance caused by the delay is bounded, that is, $\left\|d_{i \tau}(k)\right\| \leq M_{0}$, where $M_{0}$ is a positive number. Assume that the short time-varying delay does not destroy the system stability. This assumption is reasonable because a servo system is basically stable with a certain stability degree that ensures the robustness of the system to some extent. Next, tracking controllers for individual-axes (6) and a contour controller for the ENMCS are designed sequentially. We abused the notation a bit by using the same variable $d_{i}(k)$ for the exogenous disturbance in (2) and (5), and for exogenous disturbance and modeling error in (6). This should not cause confusion.

\section{Design of Tracking Controller for Individual Axis}

In this section, an ESO-based ILC tracking controller is designed for the tracking control of individual axis. And the stability is analyzed.

3.1 ESO-based ILC Tracking Controller The relative degree of the plant of individual axis is two. Thus, a second-order iterative updating law is required. Tracking errors in current trial are used to accelerate the convergence speed. The updating law is constructed to be

$$
u_{j+1}(k)=u_{j}(k)+\sum_{m=0}^{2} \Gamma_{m} e_{j}(k+m)+L e_{j+1}(k), \cdots
$$

where $j$ is an iteration index, $e_{j}$ is the system output error, and $\Gamma_{m}(m=0,1,2)$ and $L$ are the constant learning gain matrices. The overall disturbance of the system is treated as an additional state variable, $v_{i 3}(k)$, which contains the delays, the modeling error and the exogenous disturbance, that is,

$$
v_{i 3}(k)=\frac{b_{i} \tau_{i k}}{h}\left(u_{i}(k-1)-u_{i}(k)\right)+d_{i}(k)
$$

Defining

$$
\frac{v_{i 3}(k+1)-v_{i 3}(k)}{h}=w_{i}(k),
$$

the system is modified to be

$$
\left\{\begin{array}{l}
v_{i 1}(k+1)=v_{i 1}(k)+h v_{i 2}(k), \\
v_{i 2}(k+1)=v_{i 2}(k) \\
\quad+h\left(-a_{i 1} v_{i 1}(k)-a_{i 2} v_{i 2}(k)+v_{i 3}(k)+b_{i} u_{i}(k)\right), \\
v_{i 3}(k+1)=v_{i 3}(k)+h w_{i}(k), \\
r_{i}(k)=v_{i 1}(k) .
\end{array}\right.
$$

Then, a linear $\mathrm{ESO}^{(34)}$ of (10) is constructed to be

$$
\left\{\begin{array}{l}
e_{i z}(k)=z_{i 1}(k)-r_{i}(k) \\
z_{i 1}(k+1)=z_{i 1}(k)+h\left(z_{i 2}(k)-\beta_{i 1} e_{i z}(k)\right) \\
z_{i 2}(k+1)=z_{i 2}(k)+h\left(-a_{i 1} z_{i 1}(k)-a_{i 2} z_{i 2}(k)\right. \\
\left.\quad+z_{i 3}(k)-\beta_{i 2} e_{i z}(k)+q_{i} u_{i}(k)\right) \\
z_{i 3}(k+1)=z_{i 3}(k)+h\left(-\beta_{i 3} e_{i z}(k)\right)
\end{array}\right.
$$

where $e_{i z}(k)$ is the estimated error; $z_{i 1}(k), z_{i 2}(k)$, and $z_{i 3}(k)$ are the estimations of the states $v_{i 1}(k), v_{i 2}(k)$, and $v_{i 3}(k)$, respectively; $q_{i}$ is a compensating factor; and $\beta_{i 1}, \beta_{i 2}, \beta_{i 3}$ are the observer gains, which are designed to be $\beta_{i 1}=3 \omega_{i 0}, \beta_{i 2}=3 \omega_{i 0}^{2}$, $\beta_{i 3}=\omega_{i 0}^{3}$, and $\omega_{i 0}$ is the observer bandwidth ${ }^{(34)}$.

In order to reject the aperiodic disturbance, the estimation of the overall disturbance, $z_{i 3}(k)$, should be incorporated in the control input, $u_{i}(k)$, in every iteration. That is, the output of the ESO, $u_{i w j}(k)$, is a real-time feedback term, which is not stored in a memory for iteration. Then, the configuration of the ESO-based ILC control system is depicted in Fig. 4, in which

$$
\left\{\begin{aligned}
u_{i j}(k) & =u_{i(j-1)}(k)+C_{f f} E_{i(j-1)}(k)+C_{f b} E_{i j}(k), \\
u_{i w j}(k) & =-\frac{z_{i 3 j}(k)}{q_{i}} \\
u_{i j}^{f}(k) & =u_{i j}(k)+u_{i w j}(k),
\end{aligned}\right.
$$

where $E_{i j}(k)=\left[e_{i j}(k+2) \quad e_{i j}(k+1) \quad e_{i j}(k)\right]$ is a tracking error vector, $C_{f f}$ is a feedforward gain, $C_{f b}$ is a feedback gain, $u_{i w j}(k)$ is the amount of compensation of the overall disturbance, and $u_{i j}^{f}(k)$ is the final output of the controller. Assume that the ESO is well tuned and the estimated error of $v_{i 3 j}(k)$ is small enough. So,

$$
z_{i 3 j}(k) \approx v_{i 3 j}(k) \text {. }
$$




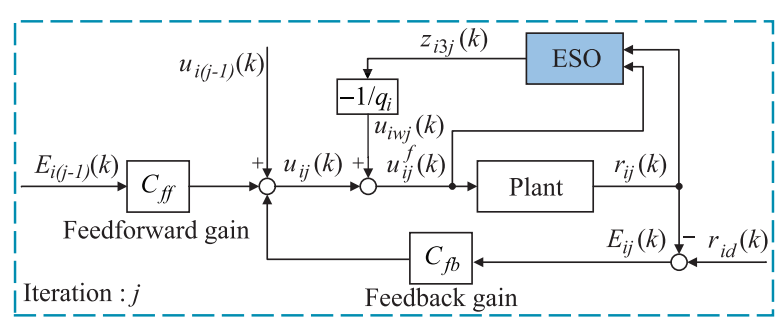

Fig. 4. Configuration of the ESO-based ILC control system for $i$-axis $(i=x, y)$

Let $q_{i}=b_{i}$. Substituting $u_{i j}^{f}(k)$ into system (6) yields

$$
\left\{\begin{aligned}
v_{i 1 j}(k+1) & =v_{i 1 j}(k)+h v_{i 2 j}(k), \\
v_{i 2 j}(k+1) & =v_{i 2 j}(k)+h\left(-a_{i 1} v_{i 1 j}(k)-a_{i 2} v_{i 2 j}(k)\right. \\
& \left.+b_{i} u_{i j}(k)\right) \\
r_{i j}(k) & =v_{i 1 j}(k) .
\end{aligned}\right.
$$

3.2 Stability Analysis Rewriting system (13) as

$$
\left\{\begin{aligned}
v_{i j}(k+1) & =A v_{i j}(k)+B u_{i j}(k) \\
r_{i j}(k) & =C v_{i j}(k)
\end{aligned}\right.
$$

where

$$
\begin{aligned}
& v_{i j}(k)=\left[\begin{array}{ll}
v_{i 1 j}(k) & v_{i 2 j}(k)
\end{array}\right]^{\mathrm{T}}, \\
& A=\left[\begin{array}{cc}
1 & h \\
-h a_{i 1} & 1-h a_{i 2}
\end{array}\right], B=\left[\begin{array}{c}
0 \\
h b_{i}
\end{array}\right], C=\left[\begin{array}{ll}
1 & 0
\end{array}\right] .
\end{aligned}
$$

In the remainder of this section, $i$ is omitted for clarity in expression in the stability analysis. Assume that there are always exist ideal input $u_{d}(k)$ and states $v_{d}(k)$ satisfy system (14) with the reference trajectory $r_{d}(k)$ when $u_{d}(k)$ is uniform boundness.

Letting the initial state of the system be $v_{j}(0)$ gives

$$
v_{j}(k)=\Phi(k) v_{j}(0)+\sum_{s=0}^{k-1} \Phi(k-s-1) B u_{j}(s), \cdots \cdots
$$

where $\Phi(k)=A^{k}$ is the state-transition matrix.

A sufficient condition for the stability of the individual-axis control system is given by the following theorem.

Theorem 1. If system (14) satisfies:

1. There is a proper gain $\Gamma_{2}$ makes $\left\|I-\Gamma_{2} C A B\right\|=\rho<1$; and

2. The initial state of every iteration is the same: $v_{j}(0)=$ $v_{0}(j=1,2, \ldots)$.

The iteration output, $r_{j}(k)$, converges to the reference trajectory, $r_{d}(k)$, on $\left[\begin{array}{ll}0 & T\end{array}\right]$ as $j \rightarrow \infty$ when the updating law (7) is applied to the system (14).

Proof. Denote

$$
\left\{\begin{array}{l}
\Delta v_{j}(k)=v_{d}(k)-v_{j}(k), \\
\Delta u_{j}(k)=u_{d}(k)-u_{j}(k), \\
e_{j}(k)=r_{d}(k)-r_{j}(k) .
\end{array}\right.
$$

Since $C B=0$, we have

$$
\left\{\begin{array}{l}
e_{j}(k+1)=C A \Delta v_{j}(k), \\
e_{j}(k+2)=C A^{2} \Delta v_{j}(k)+C A B \Delta u_{j}(k),
\end{array}\right.
$$

and

$$
\Delta u_{j+1}(k)=u_{d(j+1)}(k)-u_{j+1}(k) .
$$

Substituting (7) into (18) yields

$$
\begin{aligned}
\Delta u_{j+1}(k)= & \Delta u_{j}(k)-\sum_{m=0}^{2} \Gamma_{m} e_{j}(k+m)-L e_{j+1}(k) \\
= & \left(I-\Gamma_{2} C A B\right) \Delta u_{j}(k)-\sum_{m=0}^{2} \Gamma_{m} C A^{m} \Delta v_{j}(k) \\
& -L C \Delta v_{j+1}(k) . \cdots \ldots \ldots \ldots \ldots \ldots \ldots \ldots \ldots
\end{aligned}
$$

Taking the norms on both sides of (19) gives

$$
\begin{aligned}
\left\|\Delta u_{j+1}(k)\right\| \leq & \left\|I-\Gamma_{2} C A B\right\| \cdot\left\|\Delta u_{j}(k)\right\|+\sum_{m=0}^{2}\left\|\Gamma_{m} C A^{m}\right\| \\
& \cdot\left\|\Delta v_{j}(k)\right\|+\|L C\| \cdot\left\|\Delta v_{j+1}(k)\right\| \\
\leq & \rho\left\|\Delta u_{j}(k)\right\|+l_{1}\left\|\Delta v_{j}(k)\right\|+l_{2}\left\|\Delta v_{j+1}(k)\right\|,
\end{aligned}
$$

where

$$
l_{1}=\sum_{m=0}^{2}\left\|\Gamma_{m} C A^{m}\right\|, l_{2}=\|L C\| .
$$

Substituting (15) into (20) yields

$$
\begin{gathered}
\left\|\Delta u_{j+1}(k)\right\| \leq \rho\left\|\Delta u_{j}(k)\right\|+l_{1} \sum_{s=0}^{k-1}\|\Phi(k-s-1) B(s)\| \\
\cdot\left\|\Delta u_{j}(s)\right\|+l_{2} \sum_{s=0}^{k-1}\|\Phi(k-s-1) B(s)\| \cdot\left\|\Delta u_{j+1}(s)\right\| \\
\leq \rho\left\|\Delta u_{j}(k)\right\|+l_{1} l_{3} \sum_{s=0}^{k-1}\left\|\Delta u_{j}(s)\right\|+l_{2} l_{3} \sum_{s=0}^{k-1}\left\|\Delta u_{j+1}(s)\right\|,
\end{gathered}
$$

where

$$
l_{3}=\sup _{0 \leq s \leq k-1}\|\Phi(k-s-1) B\| .
$$

Multiplying both sides of (21) by $\lambda^{k}(0<\lambda<1)$, we have

$$
\begin{aligned}
& \lambda^{k}\left\|\Delta u_{j+1}(k)\right\| \leq \rho \lambda^{k}\left\|\Delta u_{j}(k)\right\|+l_{1} l_{3} \sum_{s=0}^{k-1} \lambda^{k-s} \lambda^{s}\left\|\Delta u_{j}(s)\right\| \\
& +l_{2} l_{3} \sum_{s=0}^{k-1} \lambda^{k-s} \lambda^{s}\left\|\Delta u_{j+1}(s)\right\| \\
& \leq \rho \lambda^{k}\left\|\Delta u_{j}(k)\right\|+l_{1} l_{3} \sum_{s=0}^{k-1} \lambda^{k-s} \sup _{0 \leq \tau \leq N}\left\{\lambda^{\tau}\left\|\Delta u_{j}(\tau)\right\|\right\} \\
& +l_{2} l_{3} \sum_{s=0}^{k-1} \lambda^{k-s} \sup _{0 \leq \tau \leq N}\left\{\lambda^{\tau}\left\|\Delta u_{j+1}(\tau)\right\|\right\} . \cdots \cdots(22)
\end{aligned}
$$

According to the definition of $\lambda$ norm:

$$
\begin{aligned}
& \qquad\|g\|_{\lambda}=\sup _{0 \leq k \leq N}\left\{\lambda^{k}\|g(k)\|\right\} \quad(0<\lambda<1), \\
& \text { and } \sum_{s=0}^{k-1} \lambda^{k-s}=\frac{\lambda\left(1-\lambda^{N}\right)}{1-\lambda} \text { yields }
\end{aligned}
$$




$$
\begin{aligned}
& \left\|\Delta u_{j+1}\right\|_{\lambda} \leq \rho\left\|\Delta u_{j}\right\|_{\lambda}+l_{1} l_{3} \frac{\lambda\left(1-\lambda^{N}\right)}{1-\lambda}\left\|\Delta u_{j}\right\|_{\lambda} \\
& \quad+l_{2} l_{3} \frac{\lambda\left(1-\lambda^{N}\right)}{1-\lambda}\left\|\Delta u_{j+1}\right\|_{\lambda} \\
& =\rho\left\|\Delta u_{j}\right\|_{\lambda}+l_{1} l_{3} l_{4}\left\|\Delta u_{j}\right\|_{\lambda}+l_{2} l_{3} l_{4}\left\|\Delta u_{j+1}\right\|_{\lambda}, \cdots
\end{aligned}
$$

where $l_{4}=\frac{\lambda\left(1-\lambda^{N}\right)}{1-\lambda}$. So,

$$
\begin{array}{r}
\left\|\Delta u_{j+1}\right\|_{\lambda} \leq \frac{\rho+l_{1} l_{3} l_{4}}{1-l_{2} l_{3} l_{4}}\left\|\Delta u_{j}\right\|_{\lambda}=\bar{\rho}\left\|\Delta u_{j}\right\|_{\lambda}, \\
1-l_{2} l_{3} l_{4}>0,
\end{array}
$$

where $\bar{\rho}=\frac{\rho+l_{1} l_{3} l_{4}}{1-l_{2} l_{3} l_{4}}$. Since $0<\rho<1$, it is possible to find a small enough $\lambda$ that satisfies $1-l_{2} l_{3} l_{4}>0$ and $\bar{\rho}<1$. Therefore, we have $\lim _{j \rightarrow \infty}\left\|\Delta u_{j}\right\|_{\lambda}=0$ and $\lim _{j \rightarrow \infty} u_{j}(k)=u_{d}(k)$. From (14), $\lim _{j \rightarrow \infty} r_{j}(k)=r_{d}(k)$ holds. This completes the proof.

\section{Design of Contour Controller}

In this section, considering that unsynchronization gives big influence on contour-tracking control, we incorporated the iteration-domain fuzzy PD and the CCC into the system to improve the contour-tracking precision. The stability analysis for incorporating CCC is demonstrated.

4.1 Integrated Contour Controller The contour error, $\varepsilon$, in a two-dimensional plane is

$$
\varepsilon=-c_{x} e_{x}+c_{y} e_{y}
$$

where $e_{x}$ and $e_{y}$ are the tracking error of $x$ - and $y$-axis, respectively; and $c_{x}$ and $c_{y}$ are coupling gains. Contour trajectories in practical applications are categorized to be linear contour, circular contour, and freeform contour, and the last one can be approximated by the first two. According to Reference (23), coupling gains for linear contour are

$$
c_{x}=\sin \theta, c_{y}=\cos \theta, \cdots
$$

and for circular contour are

$$
c_{x}=\sin \theta-\frac{e_{x}}{2 R}, c_{y}=\cos \theta+\frac{e_{y}}{2 R} .
$$

CCC couples the individual axes to be a single unit, and the contour control system is constructed as shown in Fig. 5. A PD cross-coupled controller is applied in this study. The output is $u_{c j}(k)$ and the input is contour errors, $\varepsilon_{j}(k)$. The derivative term of the PD regulator is approximated by the Euler method. Subsequently, the output is decomposed in two signals: $u_{x c j}(k)$ and $u_{y c j}(k)$, to compensate for $x$ - and $y$ axis through the individual coupling gains, respectively. So,

$$
\left\{\begin{aligned}
u_{c j}(k) & =K_{c p} \varepsilon_{j}(k)+K_{c d}\left(\varepsilon_{j}(k)-\varepsilon_{j}(k-1)\right), \\
u_{x c j}(k) & =-c_{x}(k) u_{c j}(k), \\
u_{y c j}(k) & =c_{y}(k) u_{c j}(k),
\end{aligned}\right.
$$

where $K_{c p}$ and $K_{c d}$ are proportional and differential gains of the CCC, respectively. Since the estimations of contour errors are inaccurate in the first few iteration trials, high CCC performance cannot be expected and small CCC gains are suitable. Later, large CCC gains are required to achieve satisfactory compensation effect. Thus, a fuzzy regulator is devised to provide such varying gains in the iteration domain

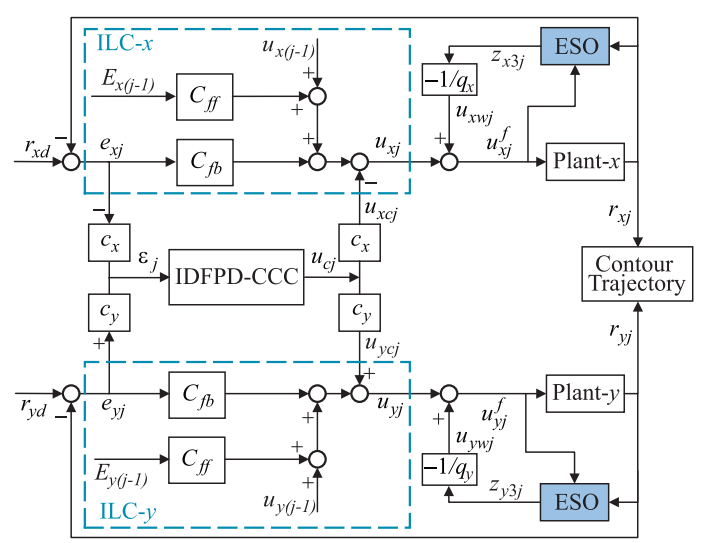

Fig. 5. Configuration of the contour control system
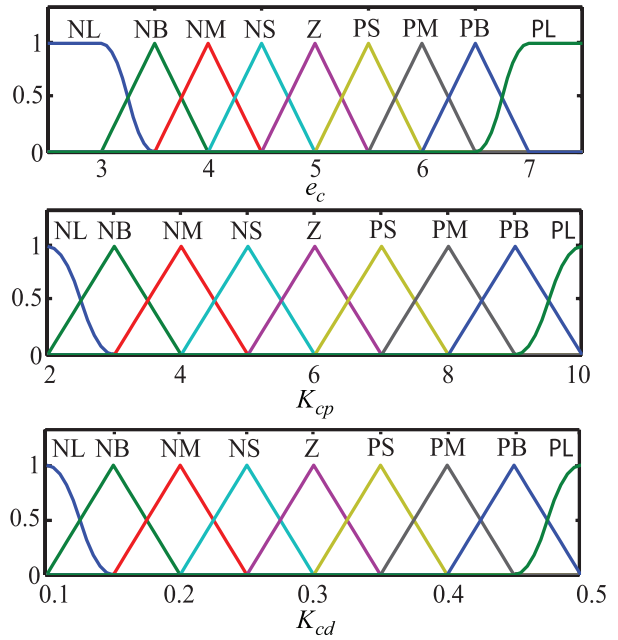

Fig. 6. Membership functions of fuzzy rules for $e_{c}, K_{c p}$, and $K_{c d}$

Table 1. Rule table of the fuzzy regulator

\begin{tabular}{c|ccccccccc}
\hline$e_{c}$ & NL & NB & NM & NS & Z & PS & PM & PB & PL \\
\hline$K_{c p}$ & PL & PB & PM & PS & Z & NS & NM & NB & NL \\
$K_{c d}$ & PL & PB & PM & PS & Z & NS & NM & NB & NL \\
\hline
\end{tabular}

and an IDFPD-CCC is derived. An input linguistic variable is chosen to be the one related to the cumulative contour error, $e_{c}=\log \left(e_{\text {sum }}\right)$, and two fuzzy output variables are $K_{c p}$ and $K_{c d}$ for two single-input, single-output fuzzy regulators. The linguistic terms are NL (Negative Large), NB (Negative Big), NM (Negative Medium), NS (Negative Small), Z (Zero), PS (Positive Small), PM (Positive Medium), PB (Positive Big), and PL (Positive Large). The membership functions are illustrated in Fig. 6. And the fuzzy rules are listed in Table 1. The fuzzy outputs are calculated using the mean value of maximum method.

Then, the output of the contour controller in the iterationdomain has two parts: the ILC output and the CCC compensation. The output of the ESO is a real-time feedback term. Therefore, the final output of the contour controller, $u_{i j}^{f}(k)$, is

$$
\left\{\begin{array}{l}
u_{i j}^{f}(k)=u_{i j}(k)+u_{i w j}(k), \\
u_{i j}(k)=u_{i(j-1)}(k)+C_{f f} E_{i(j-1)}(k)+C_{f b} E_{i j}(k)+u_{i c j}(k), \\
u_{i w j}(k)=-\frac{z_{i 3 j}(k)}{q_{i}}
\end{array}\right.
$$


4.2 Stability Analysis for Incorporating CCC The contour controller adds the amount of contour compensation, $u_{i c j}(k)$, to the individual-axis tracking controller. The amount of the compensation added to each axis was restricted in a certain range in this study, that is, $\left\|u_{i c j}(k)\right\| \leq M$, where $M$ is a positive number. Taking the compensation part into account, we rewrite (24) to be

$$
\left\|\Delta u_{j+1}(k)\right\|_{\lambda} \leq \bar{\rho}\left\|\Delta u_{j}(k)\right\|_{\lambda}+M^{\prime}
$$

where $M^{\prime}=\frac{M}{1-l_{2} l_{3} l_{4}}$. This yields

$$
\left\|\Delta u_{j}(k)\right\|_{\lambda} \leq \bar{\rho}^{j}\left\|\Delta u_{0}(k)\right\|_{\lambda}+\frac{1-\bar{\rho}^{j}}{1-\bar{\rho}} M^{\prime}
$$

Since $\bar{\rho}<1$, we can conclude that $\left\|\Delta u_{j}(k)\right\|_{\lambda}$ is bounded, and

$$
\lim _{j \rightarrow \infty}\left\|\Delta u_{j}(k)\right\|_{\lambda} \leq \frac{M^{\prime}}{1-\bar{\rho}} .
$$

(14), (15), and (16) gives

$$
\begin{aligned}
e_{j}(k)= & C v_{d}(k)-C v_{j}(k) \\
= & C\left(\Phi(k) v_{d}(0)+\sum_{s=0}^{k-1} \Phi(k-s-1) B u_{d}(s)-\Phi(k) v_{j}(0)\right. \\
& \left.-\sum_{s=0}^{k-1} \Phi(k-s-1) B u_{j}(s)\right) \\
= & C \sum_{s=0}^{k-1} \Phi(k-s-1) B\left(u_{d}(s)-u_{j}(s)\right) \\
= & C \sum_{s=0}^{k-1} \Phi(k-s-1) B \Delta u_{j}(s) . \cdots \cdots \cdots(33)
\end{aligned}
$$

Tacking the norms on both sides of (33) gives

$$
\begin{aligned}
\left\|e_{j}(k)\right\| & \leq\|C\| \cdot \sum_{s=0}^{k-1}\|\Phi(k-s-1) B\| \cdot\left\|\Delta u_{j}(s)\right\| \\
& \leq l_{5} \sum_{s=0}^{k-1}\left\|\Delta u_{j}(s)\right\|, \ldots \ldots \ldots \ldots \ldots \ldots
\end{aligned}
$$

where $l_{5}=\|C\| \cdot \sup _{0<s<k-1}\|\Phi(k-s-1) B\|$.

Multiplying both sides of (34) by $\lambda^{k}(0<\lambda<1)$ yields

$$
\begin{aligned}
\lambda^{k}\left\|e_{j}(k)\right\| & \leq l_{5} \lambda^{k} \sum_{s=0}^{k-1}\left\|\Delta u_{j}(s)\right\| \\
& =l_{5} \sum_{s=0}^{k-1} \lambda^{k-s} \lambda^{s}\left\|\Delta u_{j}(s)\right\| \\
& \leq l_{5} \sum_{s=0}^{k-1} \lambda^{k-s} \sup _{0 \leq \tau \leq N}\left\{\lambda^{\tau}\left\|\Delta u_{j}(\tau)\right\|\right\} .
\end{aligned}
$$

The definition of $\lambda$-norm provides

$$
\left\|e_{j}(k)\right\|_{\lambda} \leq l_{4} l_{5}\left\|\Delta u_{j}(k)\right\|_{\lambda}
$$

Then, according to (32), we have

$$
\lim _{j \rightarrow \infty}\left\|e_{j}(k)\right\|_{\lambda} \leq l_{4} l_{5} \frac{M^{\prime}}{1-\bar{\rho}} .
$$

Therefore, the contour control system is bounded-input, bounded-output stable. Appropriate small CCC gains should be chosen so as to avoid the breakdown of the stability of the individual axes. On the other hand, the contour error decreases as the individual-axis tracking precision increases. Thus, $M^{\prime} \rightarrow 0$ as $j \rightarrow \infty$, that is, $\lim _{j \rightarrow \infty}\left\|\Delta u_{j}(k)\right\|_{\lambda}=0$ and $\lim _{j \rightarrow \infty}\left\|e_{j}(k)\right\|_{\lambda}=0$. Fuzzy regulator is designed empirically to achieve satisfactory results.

\section{Simulation and Experimental Results}

In this section, simulations and experiments are carried out to demonstrate the feasibility and effectiveness of the ENMCS. Identifying the models of individual axes using the particle swarm optimization method in local mode yielded

$$
\left\{\begin{array}{l}
P_{x}(s)=\frac{R_{x}(s)}{U_{x}(s)}=\frac{4.41}{s(s+51.58)} \\
P_{y}(s)=\frac{R_{y}(s)}{U_{y}(s)}=\frac{4.10}{s(s+47.77)} \\
a_{x 1}=0, a_{x 2}=51.58, b_{x}=4.41 \\
a_{y 1}=0, a_{y 2}=47.77, b_{y}=4.10 .
\end{array}\right.
$$

For comparison, control is performed by four strategies:

(1) ILC approach: Individual-axis controllers are designed based on ILC without contour compensation;

(2) ILC + ESO approach: Individual-axis controllers are designed based on ILC and ESO without contour compensation;

(3) $\quad$ ILC + ESO + PD-CCC approach: Individual-axis controllers are designed based on ILC, and conventional

PD-CCC is used to compensate for the contour errors; and (4) ILC + ESO + IDFPD-CCC approach: It is the approach devised in this paper.

For the selection of the parameters of the ILC, $\Gamma_{0}, \Gamma_{1}, \Gamma_{2}$, and $L$, first, they have to satisfy Condition 1 in Theorem 1 . Second, they have also needed to adapt to the processing characteristics of the experimental carving machine, which worked at a relatively low speed. As a result, they were chosen to be

$$
\Gamma_{0}=50, \Gamma_{1}=-370, \Gamma_{2}=320, L=1 .
$$

On the other hand, the bandwidth of the ESO, $\omega_{i 0}$, had a wide range of adaptation for the ENMCS and was selected to be $\omega_{x 0}=\omega_{y 0}=100$, so as to ensure rapid estimation and compensation for the disturbance. The compensating factors, $q_{x}$ and $q_{y}$, of the ESO were set to be the same as the system parameters $b_{x}$ and $b_{y}$, respectively, that is, $q_{x}=4.41$ and $q_{y}=4.10$.

A simple check shows that Condition 1 in Theorem 1 is satisfied for

$$
\rho_{x}=0.965, \rho_{y}=0.967 .
$$

The proportional and differential gains of conventional PDCCC was empirically set to be $K_{p}=10$ and $K_{d}=0.5$, respectively, which are the maximum obtained for the fuzzy regulators.

Set $T=8 \mathrm{~s}, h=5 \mathrm{~ms}$, and the circular reference trajectory and initial states as follows:

$$
\begin{aligned}
& x_{d}=10 \cos 0.25 \pi t+150 \mathrm{~mm}, \\
& y_{d}=10 \sin 0.25 \pi t+150 \mathrm{~mm}, \quad 0 \leq t \leq T ;
\end{aligned}
$$




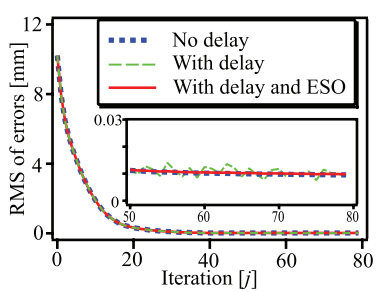

(a)

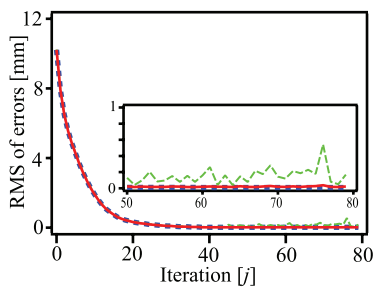

(c)

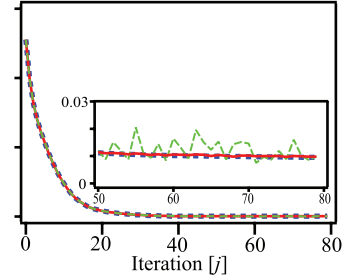

(b)

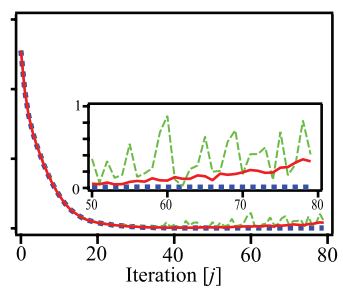

(d)
Fig. 7. The compensation of the ESO for time-varying delays ( $x$-axis). (a) Delay: 0-5 ms; (b) Delay: 0-10 ms; (c) Delay: 0-50 ms; (d) Delay: 0-100 ms

$$
\begin{aligned}
& x_{j}(0)=x_{d}^{0}=160 \mathrm{~mm}, \\
& y_{j}(0)=y_{d}^{0}=150 \mathrm{~mm} .
\end{aligned}
$$

5.1 Numerical Simulations Numerical simulations were carried out in a MATLAB environment. First, in order to demonstrate the effectiveness of the compensation of the ESO for a time-varying delay, simulations were carried out for the delay of four different lengths: (a) $0 \leq \tau_{k} \leq 5 \mathrm{~ms}$, (b) $0 \leq \tau_{k} \leq 10 \mathrm{~ms}$, (c) $0 \leq \tau_{k} \leq 50 \mathrm{~ms}$, and (d) $0 \leq \tau_{k} \leq 100 \mathrm{~ms}$. The RMS (root mean square) tracking errors for the delay of those lengths (Fig. 7) show that the tracking-control system was stable for the delay of the first three lengths, and the ESO suppressed the effect of the delay. However, the delay of the fourth length was too large that the stability of the system was destroyed. So, even though the ESO reduced the effect of the delay to some extent, the tracking error gradually became larger as iteration progressed. This shows that the ESO is effective in compensating for time-varying delays of the ENMCS, especially for a short time-varying delay. Then, an aperiodic disturbance, which is an uniform white noise signal with amplitude of 5, was added to the system in the same channel as the control input to represent the exogenous disturbance, the modeling error and the network-induced delays.

Figure 8 shows the RMS tracking errors of individual axes, where ' $(d)$ ' means that the aperiodic disturbance was added. The cumulative contour errors in the last iteration trial are shown in Fig. 9. Absolute values of the maximum contour errors, $\left|e_{\max }\right|$, and the cumulative contour errors, $e_{\text {sum }}$, are listed in Table 2. It is clear that the individual-axis controllers performed well for both disturbance rejection and tracking control. The CCC largely decreased the contour errors. More precisely, the new approach yielded $\left|e_{\max }\right|=0.0035 \mathrm{~mm}$ and $e_{\text {sum }}=1.32 \mathrm{~mm}$. The $e_{\text {sum }}$ is reduced by $92.04 \%$ than that for the ILC $(d)$ approach. The PD-CCC controller resulted in a large error in the first few iteration trials of $y$-axis (Fig. 8). The error was eliminated by the IDFPD-CCC controller effectively since coefficients of the controller were automatically regulated and set to be small at the first few iteration trials.

5.2 Experimental Verification Experiments were
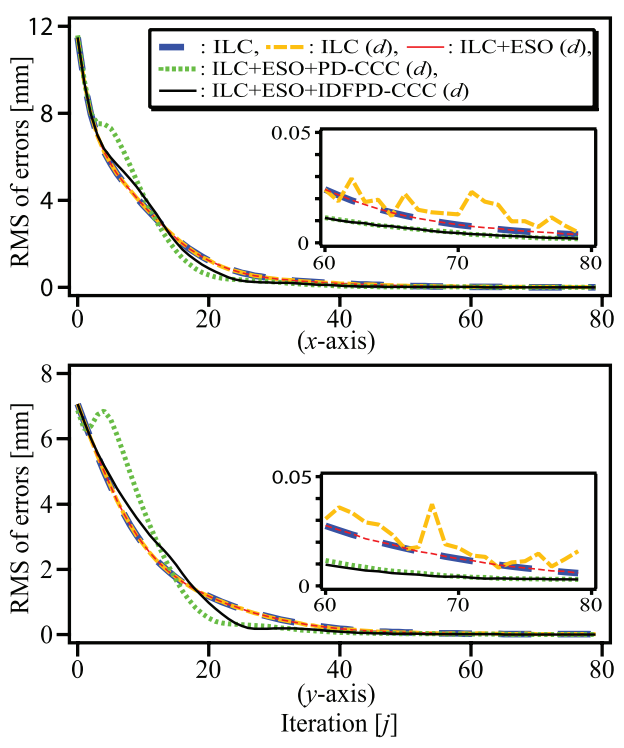

Fig. 8. RMS of tracking errors for $x$ - and $y$-axis (simulation; $(d)$ : with disturbance)

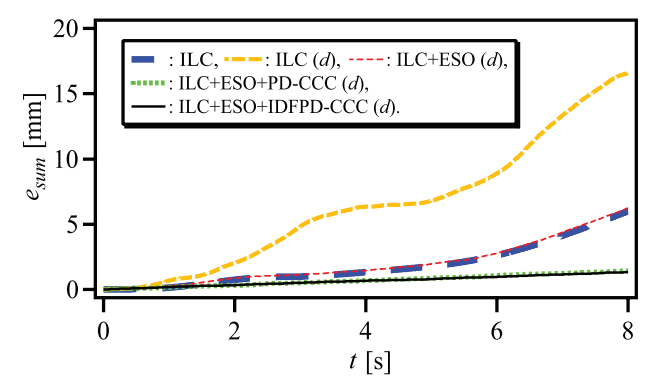

Fig. 9. Cumulative contour errors in the last iteration (simulation; $(d)$ : with disturbance)

Table 2. Contour errors of simulation results (Unit: $\mathrm{mm} ;(d)$ : with disturbance)

\begin{tabular}{ccc}
\hline Approach & $\left|e_{\max }\right|$ & $e_{\text {sum }}$ \\
\hline ILC & 0.0096 & 5.98 \\
$\operatorname{ILC}(d)$ & 0.0271 & 16.58 \\
ILC + ESO $(d)$ & 0.0115 & 6.22 \\
LC + ESO + PD-CCC $(d)$ & 0.0041 & 1.42 \\
ILC + ESO + IDFPD-CCC $(d)$ & 0.0035 & 1.32 \\
\hline
\end{tabular}

carried out using the ENMCS experimental setup. The PC runs the operating system of 64-bit Windows 10 (CPU: i36100, RAM: 4GB), and a human-computer interface program was written in $\mathrm{C}++$. The interface board is designed by a stm32 MCU and the TCP/IP protocol and CANopen protocol are developed. Recording time-points of the Ethernet transmission through timestamp, the time-varying delay, $\tau_{k}$, of every control period in one iteration trial is shown in Fig. 10, which is a short delay with $1.3 \mathrm{~ms} \leq \tau_{k} \leq 1.8 \mathrm{~ms}$. Moreover, besides the delays, an aperiodic disturbance, which is an uniform white noise signal with amplitude of 4, was added to the system to represent the exogenous disturbance and modeling error.

Individual-axis tracking errors and the cumulative contour errors are shown in Figs. 11 and 12, respectively. $\left|e_{\max }\right|$ and $e_{\text {sum }}$ of the experiments are listed in Table 3 . Note that $\left|e_{\max }\right|=0.0176 \mathrm{~mm}$ and $e_{\text {sum }}=5.73 \mathrm{~mm}$ for the new approach, in particular, $e_{\text {sum }}$ is reduced by $68.43 \%$ than that of 


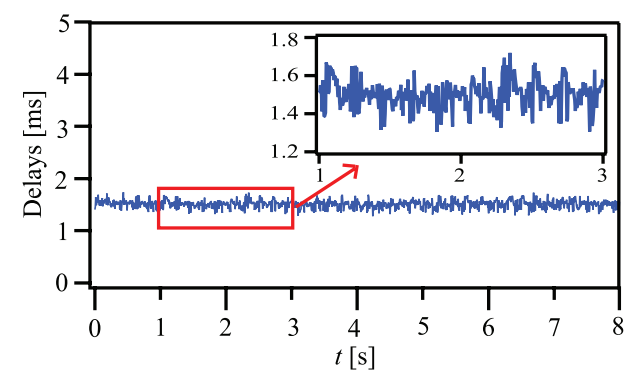

Fig. 10. Time-varying delays in one iteration trial
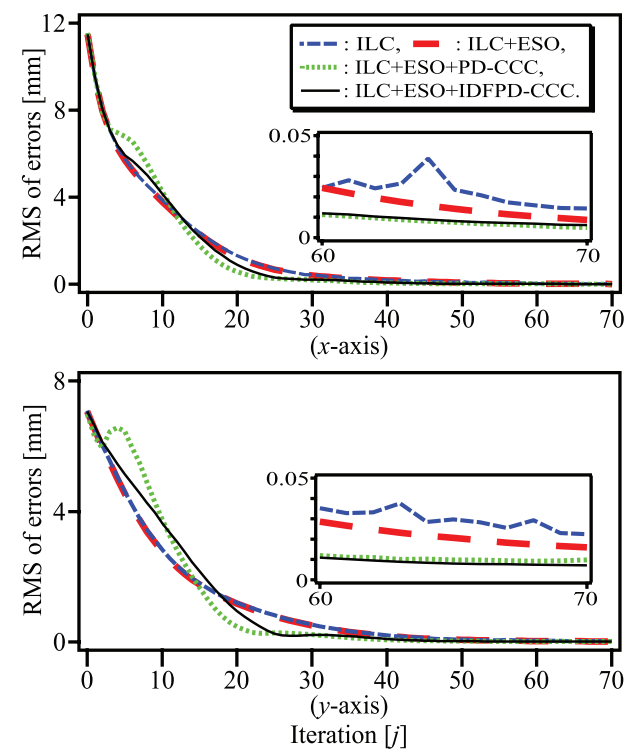

Fig. 11. RMS of tracking errors for $x$ - and $y$-axis (experiment)

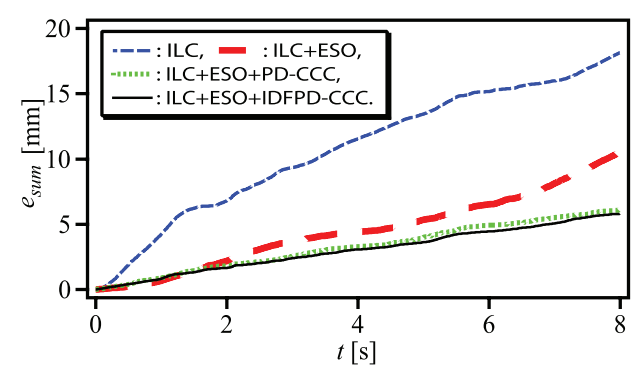

Fig. 12. Cumulative contour errors in the last iteration (experiment)

Table 3. Contour errors of experimental results (unit: $\mathrm{mm})$

\begin{tabular}{c|c|c}
\hline Approach & $\left|e_{\max }\right|$ & $e_{\text {sum }}$ \\
\hline ILC & 0.0355 & 18.15 \\
\hline ILC + ESO & 0.0257 & 10.50 \\
\hline LC + ESO + PD-CCC & 0.0207 & 6.08 \\
\hline ILC + ESO + IDFPD-CCC & 0.0176 & 5.73 \\
\hline
\end{tabular}

the ILC approach. According to the $e_{\text {sum }}$, the outputs of the fuzzy regulator, $K_{c p}$ and $K_{c d}$, are shown in Fig. 13. They are small for the first few iteration trials at the beginning of the transient response so as to avoid over compensation for individual axes. They gradually become large and converge to expected values. Similar to the simulation results, the experimental results also show the superiority of the new approach

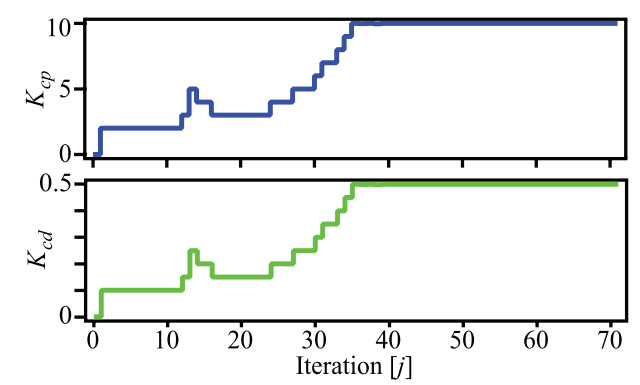

Fig. 13. $\quad K_{c p}$ and $K_{c d}$ of the fuzzy regulator (experiment)

over other methods.

Note that a contour-tracking error is the overall effect of the tracking errors of individual axes. Thus, reducing the contour error does not mean reducing the tracking error of a signal individual axis, and the $\mathrm{CCC}$ may result in a bigger tracking error for one of the individual axes. Also note that the performance of the ILC + ESO + PD-CCC and the ILC + ESO + IDFPD-CCC approaches is almost the same at the steady state, but is a little difference in the transient response. Since our goal is to achieve high-precision contour tracking control of the ENMCS, a smooth iteration process with a high steady-state precision is desirable. In this sense, the ILC + $\mathrm{ESO}+\mathrm{IDFPD}-\mathrm{CCC}$ performs better than the ILC + ESO + PD-CCC.

\section{Conclusion}

This paper presented a general-purpose Ethernet-based networked motion control system, and its contour-tracking control, which was hindered by the network-induced delays and aperiodic exogenous disturbance, was investigated when performing repetitive tasks. The delays was modeled to be bounded disturbance, and an ESO was utilized to reject the overall disturbance of the system. Then, an iterative learning controller was designed to achieve high-precision tracking control for individual axes. An IDFPD-CCC controller was devised to further improve the contour-tracking performance. The iteration-domain fuzzy regulator was utilized to obtain varying gains of the cross-coupled controller in order to avoid over compensation for individual axes in the first few iteration trials due to the tracking errors were big. Simulation and experimental results showed that the ESO-based ILC controller achieved a satisfactory individual-axis tracking performance under the aperiodic disturbance, and the IDFPD-CCC controller had made an improvement in contour tracking without degrading the control performance of individual-axis tracking. In addition, the new system is simple to establish and the approach is easy to implement, which provides a new insight and instruction for the industry application of networked motion control systems.

In this study, we mainly considered and examined the configuration of the ENMCS with a short time delay. However, an ENMCS may have a large time delay and contains mismatched disturbances. How to deal with these problems is of great significance, and will be carried out in the future.

\section{Acknowledgment}

This work was supported by the NSFC-Zhejiang Joint Fund for the Integration of Industrialization and Informatization (No.U1709213). 


\section{References}

( 1 ) J.P. Thomesse: "Fieldbus technology in industrial automation", Proceedings of the IEEE, Vol.93, No.6, pp.1073-1101 (2015)

( 2 ) M. Felser: "Real-time Ethernet-industry prospective", Proceedings of the IEEE, Vol.93, No.6, pp.1118-1129 (2005)

( 3 ) J.Y. Fei, B. Gao, and J.Y. Wen: "ARM distributed numerical control system design based on CAN-bus", Applied Mechanics and Materials, Vol.415, pp.113-116 (2013)

( 4 ) K. Erwinski, M. Paprocki, L.M. Grzesiak, K. Karwowski, and A. Wawrzak: "Application of Ethernet powerlink for communication in a linux open $\mathrm{CNC}$ system", IEEE Transactions on Industrial Electronics, Vol.60, No.2, pp.628636 (2013)

( 5 ) S.Y. Wang, J. Yang, and D. Li: "An integrated industrial Ethernet solution for the implementation of smart factory", IEEE Access, Vol.5, pp.25455-25462 (2017)

( 6 ) W.A. Zhang and L. Yu: "BIBO stability and stabilization of networked control systems with short time-varying delays", International Journal of Robust Nonlinear Control, Vol.21, pp.295-308 (2011)

( 7 ) Y. Shi, J. Huang, and B. Yu: "Robust tracking control of networked control systems: application to a networked DC motor", IEEE Transactions on Industrial Electronics, Vol.60, No.12, pp.5864-5874 (2013)

( 8 ) W.A. Zhang and L. Yu: "New approach to stabilization of networked control systems with time-varying delays", IET Control Theory \& Applications, Vol.2, No.12, pp.1094-1104 (2009)

( 9 ) Q. Li, Y. Shi, J.F. Pan, B.G. Xu, and H.X. Li: "Robust control for a networked direct-drive linear motion control system: design and experiments", Information Sciences, pp.370-371(C), pp.725-742 (2016)

(10) C.L. Lai and P.L. Hsu: "Design the remote control system with the time-delay estimator and the adaptive smith predictor", IEEE Transaction on Industrial Informatics, Vol.6, No.1, pp.73-80 (2010)

(11) G.P. Liu, J.X. Mu, D. Rees, and S.C. Chai: "Design and stability of networked control systems with random communication time delay using the modified MPC", International Journal of Control, Vol.79, No.4, pp.288-297 (2006)

(12) K. Natori and K. Ohnishi: "A design method of communication disturbance observer for time delay compensation, taking the dynamic property of network disturbance into account", IEEE Transactions on Industrial Electronics, Vol.55, No.5, pp.2152-2168 (2008)

(13) W.H. Chen, J. Yang, L. Guo, and S.H. Li: "Disturbance-observer-based control and related methods-An overview", IEEE Transactions on Industrial Electronics, Vol.63, No.2, pp.1083-1095 (2016)

(14) S.L. Chen and C.Y. Chou: "Contouring control of multi-axis motion systems for NURBS paths", IEEE Transactions on Automation Science and Engineering, Vol.13, No.2, pp.1062-1071 (2016)

(15) L. Tang and R.G. Landers: "Multiaxis contour control—-the state of the art", IEEE Transactions on Control Systems Technology, Vol.21, No.6, pp.1997$2010(2013)$

(16) C.S. Chen and L.Y. Chen: "Robust cross-coupling synchronous control by shaping position commands in multiaxes system", IEEE Transactions on Industrial Electronics, Vol.59, No.12, pp.4761-4773 (2012)

(17) N.G. Ulu, E. Ulu, and M. Cakmakci: "Design and analysis of a modular learning based cross-coupled control algorithm for multi-axis precision positioning systems", International Journal of Control Automation \& Systems, Vol.14, No.1, pp.272-281 (2016)

(18) Z.Y. Jia, J.W. Ma, D.N. Song, F.J. Wang, and W. Liu: "A review of contouring-error reduction method in multi-axis CNC machining", International Journal of Machine Tools \& Manufacture, Vol.125, pp.34-54 (2018)

(19) Y. Koren: "Cross-coupled biaxial computer control for manufacturing systems", Journal of Dynamic Systems Measurement \& Control, Vol.102, pp.265-272 (1980)

(20) D.A. Bristow, M. Tharayil, and A.G. Alleyne: "A survey of iterative learning control", IEEE Control System Magazine, Vol.26, pp.96-114 (2006)

(21) J.H. Wu, C. Liu, Z.H. Xiong, and H. Ding: "Precise contour following for biaxial systems via an A-type iterative learning cross-coupled control algorithm", International Journal of Machine Tools \& Manufacture, Vol.93, pp.10-18 (2015)

(22) W.L. Kuo, M.Y. Cheng, and C.W. Tsai: "Design of cross-coupled CMAC for contour-following - a reinforcement-based ILC approach", Aotomatika, Vol.58, No.3, pp.302-311 (2018)

(23) K.L. Barton and A.G. Alleyne: "A cross-coupled iterative learning control design for precision motion control", IEEE Transactions on Control Systems Technology, Vol.16, No.6, pp.1218-1231 (2008)

(24) J. Ling, Z. Feng, D.J. Yao, and X.H. Xiao: "Non-linear contour tracking using feedback PID and feedforward position domain cross-coupled iterative learning control", Transactions of the Institute of Measurement \& Control, Vol.40, No.6, pp.1970-1982 (2018)

(25) Y.Q. Chen and K.L. Moore: "Harnessing the nonrepetitiveness in iterative learning control", Proceedings of the 41st IEEE Conference on Decision and Control, Las Vegas, USA, pp.3350-3355 (2002)

(26) J.K. Sun and S.H. Li: "Disturbance observer based iterative learning control method for a class of systems subject to mismatched disturbances", Transactions of the Institute of Measurement \& Control, Vol.39, No.11, pp.17491760 (2016)

(27) P. Yu, M. Wu, J.H. She, K.Z. Liu, and Y. Nakanishi: "An improved equivalent-input-disturbance approach for repetitive control system with state delay and disturbance", IEEE Transactions on Industrial Electronics, Vol.65, No.1, pp.521-531 (2018)

(28) A.H.M. Sayem, Z.W. Cao, and Z.H. Man: "Model free ESO-based repetitive control for rejecting periodic and aperiodic disturbances", IEEE Transactions on Industrial Electronics, Vol.64, No.4, pp.3433-3441 (2017)

(29) J.Q. Han: "From PID to active disturbance rejection control", IEEE Transactions on Industrial Electronics, Vol.56, No.3, pp.900-906 (2009)

(30) Z. Wang and X. Jiao: "ADRC-based transient air/fuel ratio control with timevarying transport delay consideration for gasoline engines", IEEJ Transactions on Electrical \& Electronic Engineering, Vol.12, No.5, pp.S117C-S124 (2017)

(31) J.X. Wang, S.H. Li, J. Yang, B. Wu, and Q. Li: "Extended state observerbased sliding mode control for PWM-based DC-DC buck power converter systems with mismatched disturbances", IET Control Theory \& Applications, Vol.9, No.4, pp.579-586 (2015)

(32) E. Yesil, M. Guzelkaya, and I. Eksin: "Fuzzy PID controllers: An overview", The Third Triennial ETAI International Conference on Applied Automatic Systems, Skopje, Macedonia, pp.105-112 (2003)

(33) T.S. Mahmoud, M.H. Marhaban, and T.S. Hong: "ANFIS: Self-tuning fuzzy PD controller for twin rotor MIMO system", IEEJ Transactions on Electrical \& Electronic Engineering, Vol.5, No.3, pp.369-371 (2010)

(34) Z.Q. Gao: "Scaling and bandwidth-parameterization based controller tuning", Proceedings of the American Control Conference, Denver, Colorado Vol.6, pp.4989-4996 (2003)

Xiang Wu (Non-member) received the B.S. and M.S. degree in Zhejiang University of Technology, Hangzhou, China in 2012 and 2015, respectively. He is currently working toward the Ph.D. degree in the same university. His research interests include disturbance rejection and networked motion control systems.

Hui Dong (Non-member) received the M.S. and Ph.D. degree in Zhe-

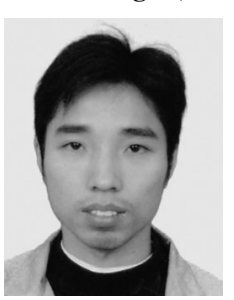
jiang University, Hangzhou, China in 2000 and 2007. respectively.He is currently a Professor in College of Information Engineering, Zhejiang University of Technology, Hangzhou, China. His current research interests include control theory, and embedded system design and its applications. 
Jinhua She (Senior Member) received the B.S. degree in engineering

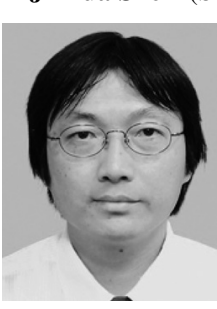
from Central South University, Changsha, China, in 1983, and the M.S. and Ph.D. degrees in engineering from Tokyo Institute of Technology, Tokyo, Japan, in 1990 and 1993, respectively. In 1993, he joined the School of Engineering, Tokyo University of Technology, where he is currently a professor. His research interests include the application of control theory, repetitive control, process control, Internet-based engineering education, and robotics. Dr. She received the IFAC (International Federation of Automatic Control) Control Engineering Practice Paper Prize in 1999 (jointly with M. Wu and M. Nakano).
Li Yu (Non-member) received the B.S. degree in control theory from

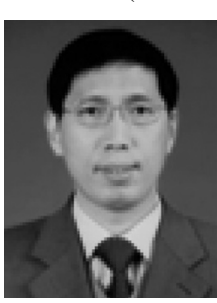

Nankai University, Tianjin, China, in 1982, and the M.S. and Ph.D. degrees from Zhejiang University, Hangzhou, China, in 1988 and 1999, respectively. $\mathrm{He}$ is currently a Professor in College of Information Engineering, Zhejiang University of Technology. He has authored or co-authored three books and over 200 journal or conference papers. His current research interests include cyber-physical systems security, wireless sensor networks, networked control systems, and 